\title{
Thermal analysis on internal and surface flaws by means of an infrared radiometer
}

\author{
by Y. Okamoto ${ }^{1}$, A. Kamoi ${ }^{1}$ and T. Ishii ${ }^{2}$ \\ 1 University of East Asia, Shimonoseki, Yamaguchi, Japan, 751-8503 \\ 2 Japan Atomic Energy Research Institute, Oarai-machi, Higashi-Ibaraki-Gun, Ibaraki, \\ Japan, 311-1394
}

\begin{abstract}
Proven non-destructive tests have been widely used to detect invisible flaws. However, those methods are not remote sensing and cannot be applied in a severe environment. A thermal technique using the infrared radiometer is useful to detect the flaw, as is allows for remote sensing. In this paper, an abnormal radiation temperature distribution of the test piece was measured to detect the internal flaw by the infrared radiometer. The detection limit of the flaws was obtained experimentally and analysed numerically by means of analysis of the conductive heat flow around the flaw. The thermal image method represents thermal and detection behaviour of surface and internal flaws using the infrared radiometer.
\end{abstract}

\section{Introduction}

The proven non-destructive tests like PT, UT and RT have been widely used to detect the surface and internal flaws like pinholes, cracks, and inciusions. These test methods are not remote sensing and cannot be applied in severe environments that are not accessible to human beings. A thermal detection technique using an infrared radiometer is useful to detect flaws, as it is a remote sensing device [1].

The principle of the infrared thermal image method is applied to detect invisible flaws by observing a distortion and transient phase difference of a surface temperature distribution using the infrared radiometer. The abnormal temperature above the flaw is generated by a different rate of thermal permeation into the medium including the flaw if the thermal properties of the medium and the flaw are different [2].

We classified the generated abnormal temperature distribution by the direction of heat flow on the surface. The vertical heating method represents the situation when the heat is injected perpendicular to the surface like sun and radiation heater. The surface temperature distribution above the flaw becomes concave and convex because of the difference of thermal permeation into the test piece and flaw. The temperature deviation of the surface over the flaw is generated by the transient change of the surface heat flux, heat transfer coefficient, thermal conductivity, and diffusivity of the medium and flaw [3].

The horizontal heating method represents conduction heat flow of the test piece which generates temperature deviations around the flaw along the surface. The surface temperature gradient around the flaw becomes non-uniform and discontinuous because contraction and expansion flows are generated in the test piece by the flaw [4].

The thermal behaviour of the test piece including the surface and internal flaw was analysed experimentally and numerically by using the thermal image method.

\section{Experimental and numerical test result of vertical test piece with internal flaw using sunlight}

An internal square flaw in a concrete wall was tested by means of solar heating. Vertical model test pieces were placed on the ground in the southern and northern direction respectively. The radiation temperature distribution of the test piece is measured by the infrared radiometer and the thermography data of the infrared radiometer are recorded in a personal computer at constant time intervals. 
Fig. 1 represents the experimental transient radiation temperature of the vertical surface of the southern and northern model pieces with internal flaws. The sun injects the heat energy into the surface of the southern wall in daytime and is cooled by atmospheric air in the evening. The convex radiation temperature distribution of the southern surface is observed from 9 óclock to 14 o'clock and becomes maximum at noon, as shown in Fig.1a. As the solar heat flux decreases in the afternoon, the heated surface of the tested piece releases the heat to the atmospheric air. The radiation temperature distribution of the southern surface becomes concave in the evening and is cooled by radiation and convection to the atmosphere. As shown in Fig.1b, the temperature of the northern wall is similar to that of the southern wall. But the temperature rise of the northern wall is smaller than that of the southern wall.

Fig. 2 shows the transient radiation temperature difference of the vertical southern and northern wall surface $\Delta T_{c}^{\prime}=T_{c^{\prime}}-T_{s^{\prime}}$, where $T_{c^{\prime}}$ is the surface radiation temperature above the center of the internal flaw and $T_{s}$ is that of the surface without the internal flaw. The temperature difference $\Delta T_{c}$ ' increases before noon and becomes maximum at noon. And $\Delta T_{c}$ ' decreases in the afternoon and becomes negative in the evening. The temperature difference of the northern wall $\Delta \mathrm{T}_{\mathrm{c}}{ }^{\prime}$ is smaller than that of the southern wall (see Fig. 2-b).

\section{Experimental and numerical results of test piece with internal flaw by using artificial heater}

When an artificial heater is used for the flaw test piece the heat flows either vertically or horizontally with respect to the surface. Fig. 3 shows an analytical two-dimensional model of vertical and horizontal heating for our numerical calculation.

The vertical heating method means that the heat flows normally to the surface. Side and back walls of the internal square flaw are insulated and the temperature gradient normal to the walls becomes zero. The heat injected on the surface is transmitted to the inside space by heat conduction. The horizontal heating generates heat flows parallel to the direction of the surface due to thermal conduction. Temperatures of right and left walls are kept constant at $T_{1}$ and $T_{2}$, respectively.

Fig. 4 shows the test apparatus for the vertical and horizontal heating methods [3]. The test piece with surface and internal flaws is heated by the electrical lamp and electric resistance heaters. The transient radiation temperature distribution of the test piece generated by the vertical heating method is measured by the $\mathrm{Hg}-\mathrm{Cd}-\mathrm{Te}$ infrared radiometer. When horizontal heating is applied, one side of the test piece is connected to the electric heater and the other side to a water cooler. The heat flows by thermal conduction along the surface from the heater to the cooler. The infrared radiometer is used to detect the location of the discontinuous and polygonal temperature distribution.

Fig. 5 shows the radiation temperature distribution of the surface of the acrylic test pieces with internal air and water flaws during vertical heating. As the thermal conductivity of the acrylic test piece $\lambda_{A}$ is larger than that of the air flaw $\lambda_{B}$, the conduction heat mainly flows in the acrylic test piece with the air flaw. The temperature distribution above the flaw becomes convex because of the contraction of flow in the acrylic test piece, as shown in Fig. 5-a. The temperature distribution of the surface with the water flaw becomes concave because of the expansion flow in the acrylic test piece with the water flaw, as shown in Fig. 5 b.

Fig. 6 shows the transient temperatures $T_{c}, T_{s}$ and the temperature difference $\Delta T_{c}$ of the acrylic test piece with internal air and water flaws. The surface temperature $T_{c}$ above the air flaw is larger than that without the flaw $T_{s}$, as shown in Fig. 6a. The temperature difference $\Delta T_{c}=T_{c}-T_{s}$ is positive and gradually increases. The surface radiation temperatures above the after flaw $T_{c}$ is smaller than that without the flaw $T_{s}$, as shown in Fig 6-b. Temperature difference $\Delta \mathrm{T}_{\mathrm{c}}$ is negative and gradually increases. It is remarked that the surface temperatures and its difference with air and water flaws are not equal tothe difference of the thermal conductivies $\lambda_{A}$ and $\lambda_{B}$. 
Table 1 represents the summarised result of the heat flow and generated temperature which depends on the difference between the thermal conductivity of the test piece and of the internal flaw. The heat flow pattern and generated temperature difference are mainiy determined by the difference of the thermal conductivities of the test piece and internal flaws $\lambda_{A}$ and $\lambda_{B}$ as shown in the table. It is important that transient heat flow and generated temperatures during the heating process become reverse in case of cooling process.

The temperature signal and shape above the flaw during horizontal heating are generated by the conduction heat flows around the flaw along the surface. The temperature gradient around the flaw becomes non-uniform and stepwise, because contraction and expansion flows are generated in the test piece with the flaw.

Fig. 7 shows the surface temperature distribution of the acrylic test pieces with internal air and water flaws by applying the horizontal heating method. The polygonal temperature gradient above the air cavity flaw becomes larger than that without the flaw, because the contraction heat flows along the acrylic plate with the air cavity flaw, as shown in Fig. $7 a$. The polygonal temperature gradient above the water cavity flaw becomes smaller than that without the cavity flaw, because the expansion heat flows along the acrylic plate with the water flaw, as shown in Fig. 7b.

Table 2 shows both the experimental and numerical temperature differences of surface and internal flaws $\Delta T_{c}$ by applying horizontal heating, as shown in Fig. 7. Upper data are obtained by experiment while data below them (in parenthesis) are numerical results. The polygonal temperature difference $\Delta T_{c}$ becomes smaller with increase in the width and decrease in the depth. Experimental and numerical temperature differences are similar to each other.

\section{Conclusion}

The thermal property around the surface and internal flaw was analyzed numerically and experimentally by using infrared thermography.

The generated abnormal temperature distribution is classified by vertical or horizontal heating directions of the surface.

When the heat is applied normal to the direction of the surface, the surface temperature distribution above the flaw becomes concave and convex in this vertical heating method.

A polygonal temperature gradient is generated by the conduction of heat around the flaw along the surface by applying the horizontal heating method, because contraction and expansion heat flows are generated in the test piece across the flaw.

Thermographic imaging is useful to analyse heat transfer mechanisms around the flaw and to evaluate detection limits for surface and internal flaws.

\section{REFERENCES}

[1] OKAMOTO (Y.), ISHII (T.) et al. - Remote-sensing thermal image method. Tokyo, Corona publisher, 1995, p. 1-173.

[2] OKAMOTO (Y.), INAGAKI (T.) et al. - Remote-sensing study of detection of flaws in structural material. Thermosense 14, SPIE Proc.1933, 1993, p. 215-224.

[3] OKAMOTO (Y.), ISHII (T.) et al. - Detection limit of flaw in material using infrared radiometer influence on direction of heat flow. Japan Soc. of NDT, 41, 7,1992, p. 398-403.

[4] OKAMOTO (Y.), INAGAKI (T.) et al. - Detection of non-uniform space in material using thermal image method. Bull. Japan Soc. of Mech. Engineering. B60, 573,1994, p. 238-245. 

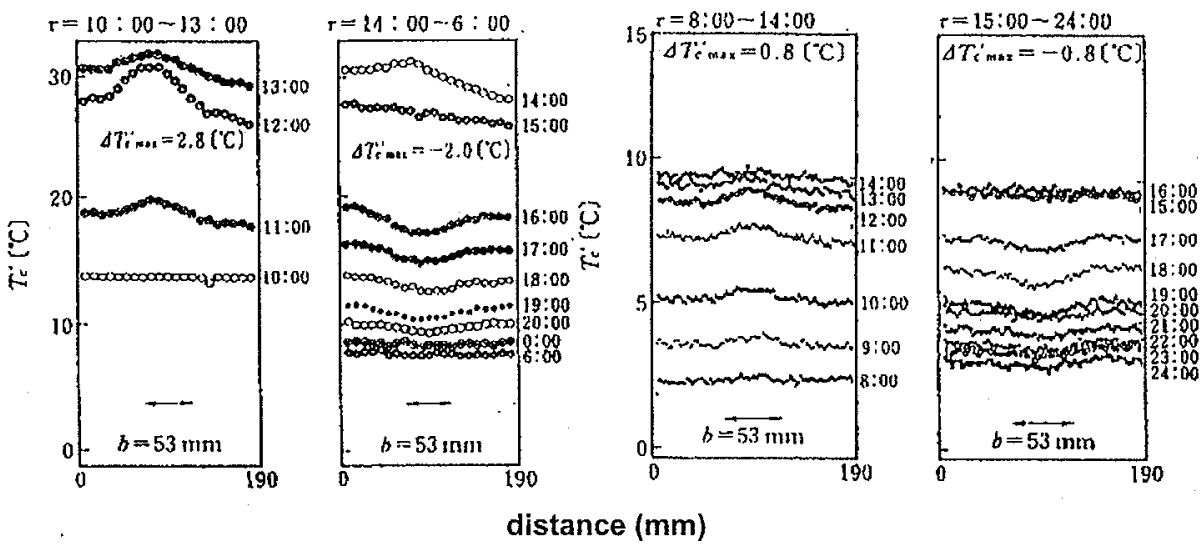

a) southern wall

b) northern wall

Fig.1. Experimental transient radiation temperature of vertical wall surface

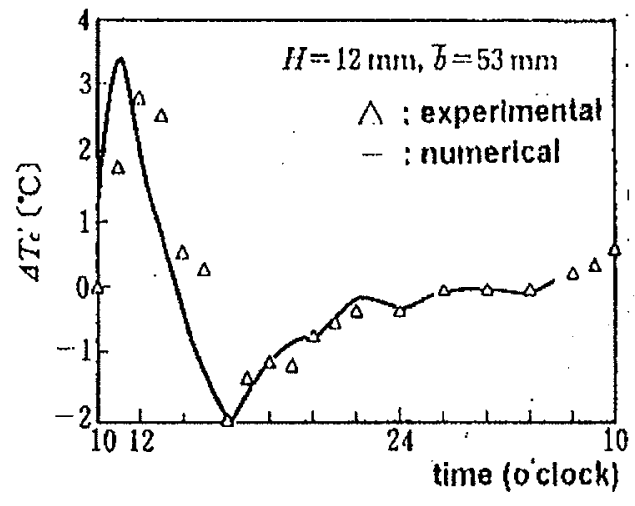

a) southern wall

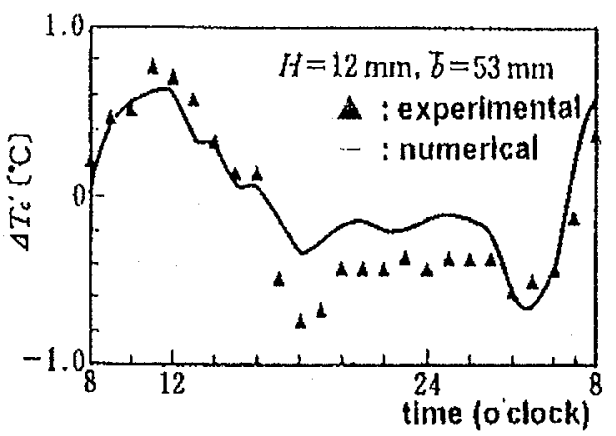

b) northern wall

Fig. 2. Transient radiation temperature difference of vertical wall surface

heat flux

$00000: 00000$

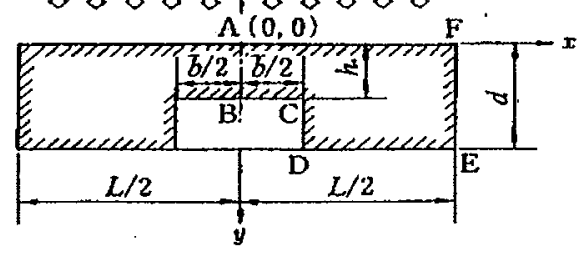

heat flow

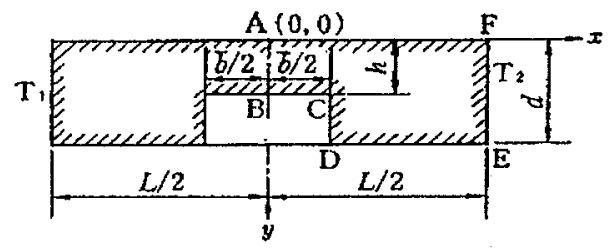

Fig. 3. Analytical model for vertical (left, a) and horizontal (right, b) heating 

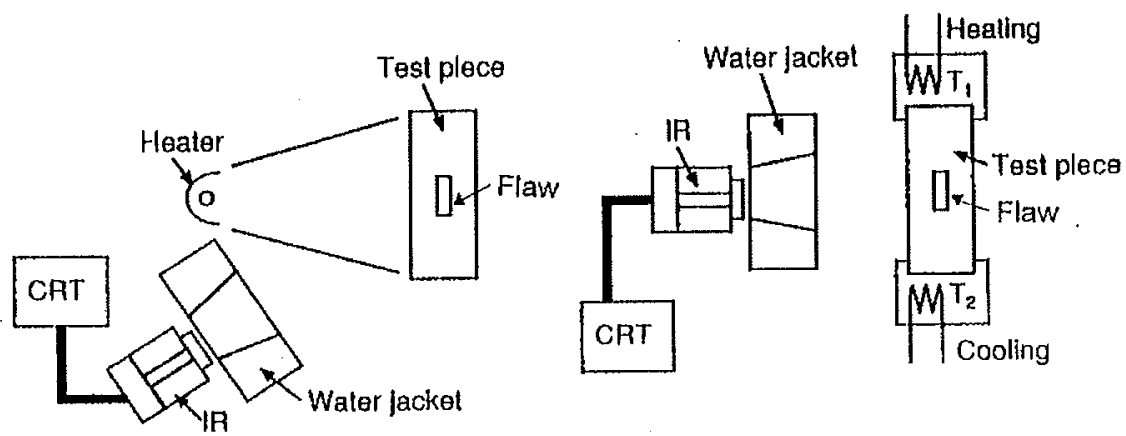

Fig. 4. Test apparatus for vertical (left, a) and horizontal (right, b) heating method

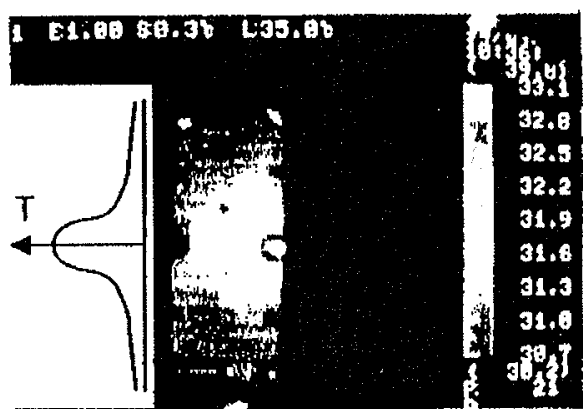

a) air flaw

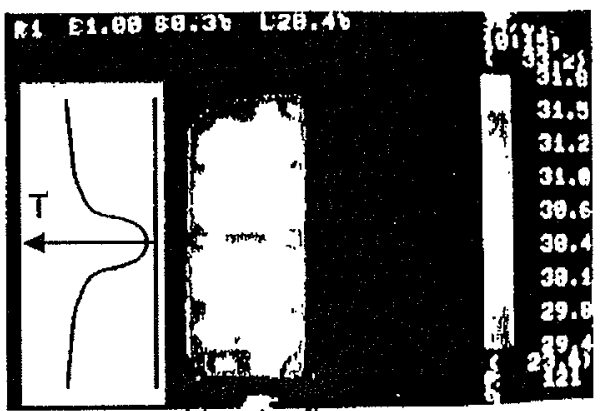

b) water llaw

Fig. 5. Radiation temperature distribution
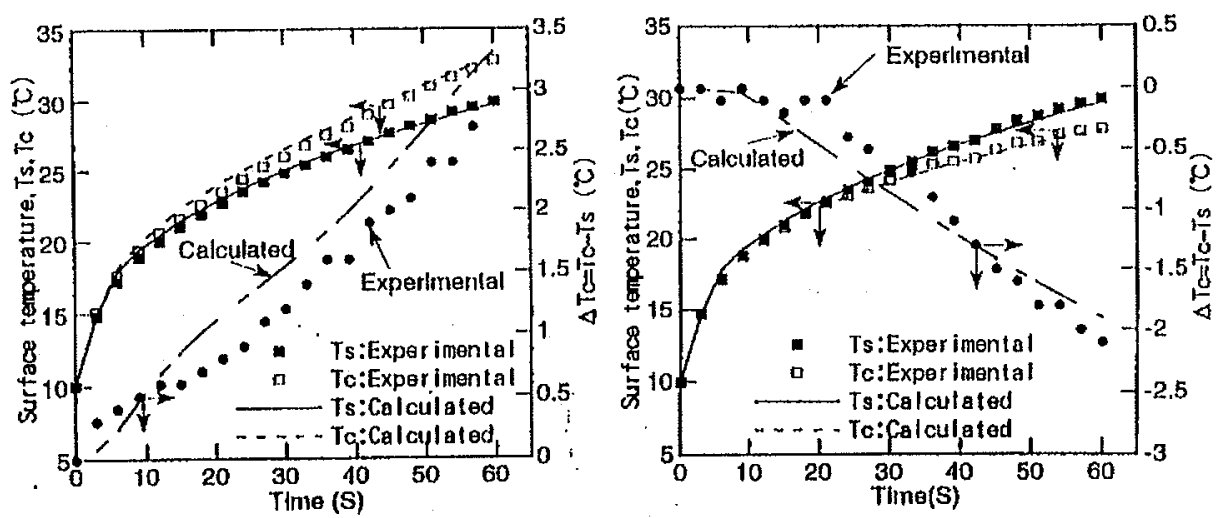

Fig. 6. Transient temperature and its difference of acrylic test piece with internal flaw (left, a: air flaw; right, b: water flaw) 
http://dx.doi.org/10.21611/qirt.1998.009

Table 1. Heat flow and generated temperature depending on thermal conductivity

\begin{tabular}{|c|c|c|c|c|}
\hline Thermal conductivity & \multicolumn{2}{|c|}{$\lambda_{\mathrm{A}}<\lambda_{\mathrm{B}}$} & \multicolumn{2}{|c|}{$\lambda_{A}>\lambda_{B}$} \\
\hline Heat transfer & heating & cooling & heating & Cooling \\
\hline Heat flow & expansion & contraction & contraction & expansion \\
\hline $\begin{array}{l}\text { Temperature } \\
\text { distribution }\end{array}$ & convex & concave & concave & convex \\
\hline Temperature difference & positive & negatlve & negative & positive \\
\hline Heat flow pattern & & $\frac{1}{4}$ & $\frac{17}{4)^{2}}$ & \\
\hline
\end{tabular}

$\lambda$. $A$; Thermal conductivity of test piece

$\lambda_{B}$; Thermal conductivity of air and water flaw
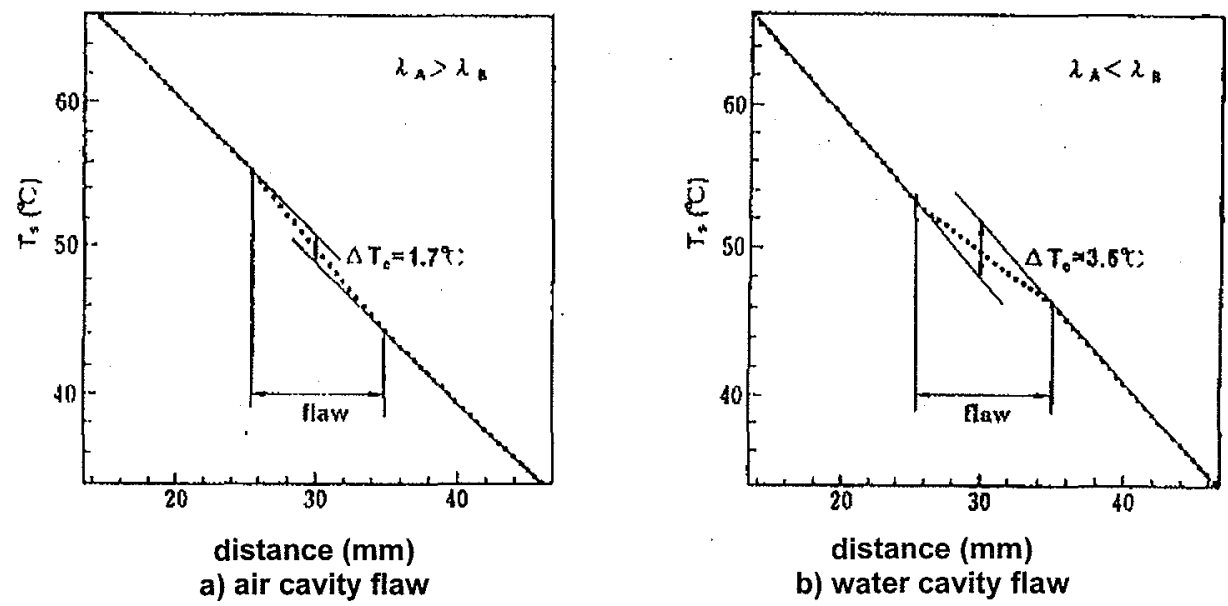

Fig. 7. Surface temperature distribution of acrylic test piece by horizontal heating

Table 2. Experimental and numerical (given in brackets) temperature difference of surface and internal flaws by horizontal heating

a) internal flaw

\begin{tabular}{c|c|c|c}
\hline \multirow{2}{*}{$\begin{array}{c}\text { width b } \\
(\mathrm{mm})\end{array}$} & \multicolumn{3}{|c}{ Depth $\mathrm{h}(\mathrm{mm})$} \\
\cline { 2 - 4 } & 1.0 & 2.0 & 5.0 \\
\hline \multirow{2}{*}{1.0} & 0.40 & 0.55 & 1.0 \\
& $(0.4)$ & $(0.6)$ & $(1.0)$ \\
\hline \multirow{2}{*}{2.0} & 0.29 & 0.39 & 0.66 \\
& $(0.3)$ & $(0.4)$ & $(0.8)$ \\
\hline \multirow{2}{*}{3.0} & 0.23 & 0.30 & 0.50 \\
& $(0.2)$ & $(0.3)$ & $(0.5)$ \\
\hline \multirow{2}{*}{4.0} & 0.19 & 0.24 & 0.41 \\
& $(0.1)$ & $(0.2)$ & $(0.3)$ \\
\hline
\end{tabular}

b) surface flaw

\begin{tabular}{c|c|c|c}
\hline \multirow{2}{*}{$\begin{array}{c}\text { width b } \\
(\mathrm{mm})\end{array}$} & \multicolumn{3}{|c}{ Depth $\mathrm{h}(\mathrm{mm})$} \\
\cline { 2 - 4 } & 1.0 & 2.0 & 5.0 \\
\hline \multirow{2}{*}{1.0} & 0.50 & 0.63 & 1.0 \\
& $(0.4)$ & $(0.8)$ & $(1.0)$ \\
\hline \multirow{2}{*}{2.0} & 0.43 & 0.50 & 0.73 \\
& $(0.2)$ & $(0.4)$ & $(0.6)$ \\
\hline \multirow{2}{*}{3.0} & 0.36 & 0.42 & 0.59 \\
& $(0.2)$ & $(0.4)$ & $(0.6)$ \\
\hline \multirow{2}{*}{4.0} & 0.30 & 0.35 & 0.49 \\
& $(0.1)$ & $(0.2)$ & $(0.6)$ \\
\hline
\end{tabular}

